\title{
Assessment of a Cognitive-Motor Training Program in Adults at Risk for Developing Dementia
}

\author{
Holly V. Echlin, Msc, Diana J. Gorbet, PhD, Lauren E. Sergio, PhD \\ School of Kinesiology and Health Science, Faculty of Health, York University, Toronto, ON, Canada \\ https://doi.org/10.5770/cgj.23.394
}

\begin{abstract}
\section{Background}

With the prevalence of dementia increasing each year, pre-clinically implemented therapeutic interventions are needed. It has been suggested that cascading neural network failures may bring on behavioural deficits associated with Alzheimer's disease.
\end{abstract}

\section{Methods}

Previously we have shown that cognitive-motor integration (CMI) training in adults with cognitive impairments generalized to improved global cognitive and activities of daily living scores. Here we employ a novel movement control-based training approach involving CMI rather than traditional cognition-only brain training. We hypothesized that such training would stimulate widespread neural networks and enhance rule-based visuomotor ability in at-risk individuals.

\section{Results}

We observed a significant improvement in bimanual coordination in the at-risk training group. We also observed significant decreases in movement variability for the most complex CMI condition in the at-risk and healthy training groups.

\section{Conclusions}

These data suggest that integrating cognition into action in a training intervention may be effective at strengthening vulnerable brain networks in asymptomatic adults at risk for developing dementia.

Key words: movement, sensorimotor integration, training, dementia risk, cognition, coordination

\section{INTRODUCTION}

In recent years, a consensus has emerged that research on non-pharmaceutical intervention strategies to prevent functional decline in dementia should focus on preclinical disease stages. ${ }^{(1)}$ A diagnosis of probable Alzheimer's disease (AD), the most common form of dementia, ${ }^{(2-4)}$ can be made only after clinical symptoms appear and significant damage to the brain has already occurred. ${ }^{(3)}$ With current clinical diagnostic techniques, it is especially difficult to detect when individuals may be in preclinical stages because behavioural symptoms are mostly absent. In this context, low-cost, non-invasive, and objective pre-clinically implemented intervention strategies would be ideal to offset this increasing health-care burden. Further, there is presently a shift away from single-domain interventions, like cognitive training (CT), to integrated, multi-domain approaches which may lead to generalizable improvements to brain health. Three recent insights drive this shift: i) pathological changes associated with dementia start years/decades before the onset of clinical symptoms; ${ }^{(3,4)}$ ii) the earliest changes appear to be an altered functionality of large brain-wide neural networks rather than atrophy in specific regions (i.e., medial-temporal area), ${ }^{(5,6)}$ iii) using CT alone does not typically produce the desired generalizability in global cognitive status or functional ability in populations with early $\mathrm{AD}(\mathrm{eAD}){ }^{(7)}$

One form of multi-domain intervention integrates exercises that require both cognition and movement. Goal-directed movements can generally be described as standard or nonstandard. While many movements made throughout our day involve simple, standard interactions in which the guiding visual information is also the goal of the movement (like reaching for a pencil), an increasingly technology-driven world has introduced many situations which require non-standard, indirect interactions. In non-standard interactions, guiding visual stimuli are not in the same spatial location as the goal of the movement, and require the learning and use of rules (implicit or explicit). These may be considered multi-domain in nature as they integrate cognition and action. Accurately performed movements involving cognitive-motor integration (CMI) require healthy white matter (WM) tracts that provide large-scale brain network communication between frontal, parietal, and subcortical brain regions. ${ }^{(5,8-10)}$

While higher-order motor deficits (e.g., apraxia) exist in late-stage $\mathrm{AD}$, individuals with $\mathrm{MCI}$ or eAD do not typically have difficulty with tasks that require a standard/direct interaction. ${ }^{(11,12)}$ However, we have previously observed that adults with $\mathrm{MCI}$, eAD, and asymptomatic adults at risk for 
dementia were impaired when asked to perform non-standard tasks requiring CMI. ${ }^{(11-14)}$ Further, we observed a relationship between the level of cognitive-motor impairment and large-scale brain network function as reflected by both WM track integrity ${ }^{(5)}$ and resting state abnormalities. ${ }^{(15)}$ Reportedly, WM disruptions are particularly characteristic of preclinical stages of AD. ${ }^{(16-18)}$ Damage to WM tracts within $\mathrm{AD}$-vulnerable networks results in decreased overall brain processing capability which reduces general performance ability on complex tasks. ${ }^{(19)}$

Previous preliminary research from our group and others has demonstrated that cognitive-motor training can improve global cognitive scores in adults with eAD and MCI..$^{(20,21)}$ These findings suggest that, since motor and cognitive processes are performed in the brain simultaneously, it would be advantageous to train them simultaneously, as well. Here, these concepts will be incorporated into a decline-prevention strategy by examining the efficacy of how a tablet-based, 16-week movement control-focused behavioural intervention program may influence cognitive, motor, and visuomotor skill performance in those who are at risk of developing dementia. Given the relevance of visuomotor skill to daily life functioning (e.g., driving), ${ }^{(2)}$ we specifically focused on a workingaged population with known familial risk for dementia. We reasoned that, if a visuomotor intervention were introduced preclinically, it would enable vulnerable brain networks to be strengthened before decline could begin, and would offer insights about decline prevention in at-risk adults. We hypothesized that: 1) the at-risk groups will perform worse than both healthy control groups on the CMI and motor measures at initial assessment; 2) all training groups will improve over time on the non-standard intervention task; and 3) the at-risk training group will demonstrate greater improvement over time on the CMI and motor assessments compared to the at-risk control group.

\section{METHODS}

\section{Participants}

For this study, 23 adults aged 50-71 were recruited from the community and placed into an at-risk $(\mathrm{n}=10 ; 6$ female and 4 male) or healthy control group ( $\mathrm{n}=13 ; 12$ female and 1 male), based on having reported a maternal history $(17,23,24)$ or multiple family members ${ }^{(18,25-28)}$ with dementia (since these seem to be highly indicative of risk status in the absence of genetic testing for AD) or no family history of dementia, respectively (Table 1). Participants in both groups

TABLE 1.

Participant N per Risk Category

\begin{tabular}{ccc}
\hline Sex/Category & $\begin{array}{c}\text { At-Risk } \\
\text { (training) }\end{array}$ & $\begin{array}{c}\text { Not At-Risk } \\
\text { (training) }\end{array}$ \\
\hline Male & $4(1)$ & $1(0)$ \\
Female & $6(4)$ & $12(6)$ \\
\hline
\end{tabular}

were randomly split into a training or no-training group. This study was approved by the Human Participants Review Sub-Committee of York University's Ethics Review Board.

\section{Measures}

\section{Demographic Questionnaire}

Participants were given a demographic questionnaire to obtain information about family history of dementia, age, sex, ethnicity, level of education, activity level, and video-game/ tablet experience. Exclusion criteria included health-related factors that could interfere with the study design such as a diagnosis of a neurological disorder, cognitive impairment, moderate/severe head injury or vision/upper limb impairment.

\section{Dementia Rating Scale-2 (DRS-2)}

The Dementia Rating Scale-2(29) is used to assess mental status and symptom severity in those with suspected dementia. The test has five subscales: attention, initiation/perseveration, construction, conceptualization, and memory. The entire test is out of 144, and a higher score is indicative of better cognitive health and a lack of symptoms of dementia.

\section{Cognitive-Motor Performance Assessment}

A visuomotor task that incorporated explicit and implicit rules for successful completion was used to assess cognitivemotor integration ability. ${ }^{(20)}$ The assessment comprised four conditions: 1) direct (slide the finger to a presented target on a touchscreen), 2) feedback reversal (move in the opposite direction to direct the cursor to the target [e.g., slide finger up to move cursor down]), 3) plane change (view targets on a vertically-placed touchscreen, but slide finger across a linked horizontally-placed touchscreen, much like one would use a touch pad on a laptop computer), and 4) plane change reversal conditions (a combination of $2 \& 3$, see Figures 1 and 2 ). The kinematic outcome variables summarized the profile of the movement based on timing (movement time, reaction time, and peak velocity of the movement), and the accuracy (absolute endpoint error) and precision (variable endpoint error) of movement execution (Appendix A).

\section{Bimanual Coordination Task}

The Bimanual Coordination Task ${ }^{(30)}$ is a timed task that involves switching between left and right hands to serially pick up a lever and move a washer onto a peg (see Figure 3).

\section{Skilled Performance (CMI) Intervention}

The intervention protocol used a video game which required the user to slide their finger to intercept moving targets on a touchscreen. Its standard computer tablet presentation was modified to require CMI (see Figure 4). This task had similar CMI principles used by the cognitive-motor assessment task, but this intervention task required more cognitive processing/ flexibility in that there were more dynamic and variable exercises presented. For example, there was a no-go component whereby a particular symbol required cognitive processing 
to inhibit movements, rather than moving to all the other objects to intercept them as they flew across the screen. This task was done in three different conditions, including the Direct, Plane Change, and Plane Change Reversal, and was played in either a practice (lack of no-go targets, set time of $60 \mathrm{sec}$ regardless of performance) or challenging mode (no-go targets, game ended when either a no-go target was touched, or three targets were missed before dropping to bottom of screen).

\section{Procedure}

All participants were asked to give written informed consent before commencing with this intervention study. The training groups did a 16-week training program in which they played the video-game that required CMI. This training was performed at home for $30 \mathrm{~min}$, twice a week, over four months, for a total of 32 sessions. The no-training groups simply did 1 hour of crosswords/puzzles from home each week. Participants were given a pre-test battery made up of the aforementioned cognitive, motor, and visuomotor measures, followed by the intervention and then the post-test battery comprising all of the same pre-test measures. During training, participants played both the practice and challenging versions in Direct,
Plane Change, and Plane Change Reversal conditions, three times each per condition.

\section{Design and Analyses}

We performed repeated measures analysis of variance tests to assess whether there was a difference in CMI ability, fine-motor skill, and cognitive status from baseline to post-intervention sessions with group and training as main factors. Paired and independent non-parametric tests were also performed. For the CMI task, an overall end point error composite variable was created by combining absolute and variable error z-scores based on the mean of both healthy control groups. Similarly, a composite timing score was calculated by combining z-scores (based on the means of both healthy control groups) for reaction time, full movement time, and the inverse of peak velocity.

To assess video-game score changes during training, data were grouped into 4 blocks (i.e., average scores of each month of training), and a repeated measures ANOVA was used to assess the progression of scores over time. The statistical analysis of data was done using SPSS statistical software (SPSS 24, IBM). The rejection level for all analyses were set at $p=.05$. a) Direct

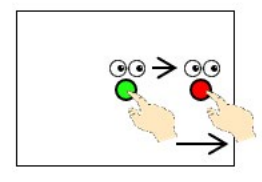

b) Feedback Reversal

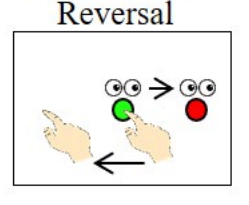

c) Plane Change

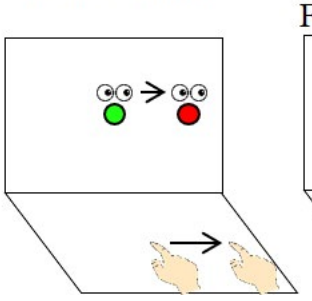

d) Plane Change / Feedback Reversal

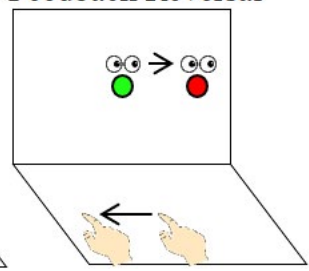

FIGURE 1. Graphic of the computer-based visuomotor conditions in the cognitive-motor task. The task requires finger-sliding movements on a touchscreen from a central target to one of four peripheral targets. The green circle denotes the center, or home, target in which all movements begin, and the lighter eye and hand symbols denote the starting position for each trial. The red circle represents the peripheral target (which appears randomly to either $90^{\circ}$ to top, bottom, left, or right of centre), and the peripheral eye and hand symbols represent the instructed eye/hand movements for the task. a) Direct (standard interaction) condition involves simple finger-sliding movements to peripheral targets with hand and eye movements on the same screen. b) Feedback Reversal (nonstandard interaction) condition incorporates a $180^{\circ}$ feedback reversal into the task. c) Plane Change (nonstandard interaction) condition involves a plane dissociation between guiding visual stimuli and hand movements. d) Plane Change Reversal (nonstandard) condition incorporates both the spatially dissociated planes between eye and hand movements, with a $180^{\circ}$ feedback reversal as well. All nonstandard tasks described here also require CMI.
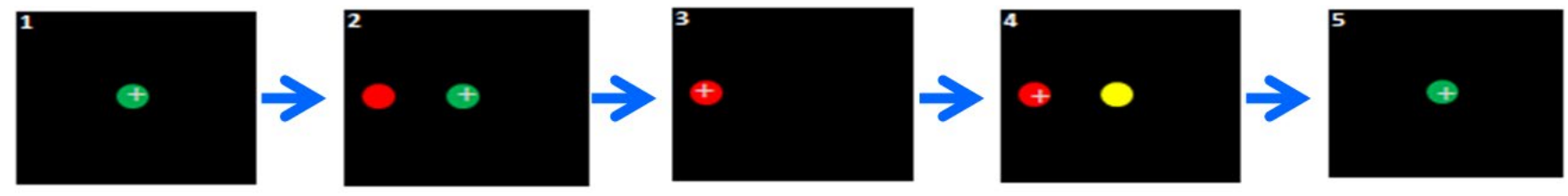

FIGURE 2. Sequence of events during one trial of the Cognitive-motor task. The green circle in the centre of box 1 denotes the home target, where all movements begin. This target changes from yellow to green to signify that the software can detect the presence of a participant's finger. After $2,000 \mathrm{~ms}$, a red peripheral target appears in one of four directions $\left(90^{\circ}\right.$ to the top, bottom, left or right of the centre target) which signifies the cue for participants to begin their finger-sliding movement toward the peripheral target. Once the participant has reached the peripheral target and remained there for an inter-trial interval of 2,000 $\mathrm{ms}$, the yellow centre home target reappears, signaling the end of the previous trial and initiation of the following trial. 


\section{RESULTS}

\section{Demographic Characteristics}

Participants were mostly female (female: 18 , male: 5 ), and Caucasian $(74 \%)$, with an average age of 62.2 years $(\mathrm{SD}=6.6)$ and an average of 16.4 years of formal education $(\mathrm{SD}=1.8)$.

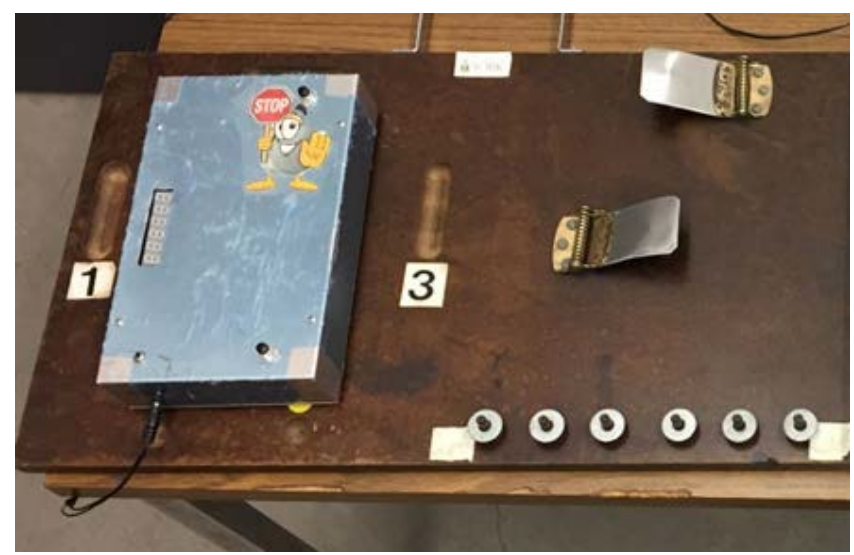

FIGURE 3. Bimanual Coordination Task. The board was placed in front of the participants on a table at approximately hip height. The six equally spaced pegs along the bottom edge of the board closest to the participants held all 12 washers which were evenly stacked in pairs. Towards the middle of the board were two more pegs, both covered by spring-loaded, hinged metal levers. The first lever closer to the participant was $18 \mathrm{~cm}$ from the bottom edge and the second lever was $32 \mathrm{~cm}$ from the bottom edge of the board. To begin, participants pressed a start button on the left side of the board and lifted the closest lever with their left hand to reveal a peg; next, their right hand moved a washer from the bottom of the board to the peg they just revealed. These steps were repeated using alternating hand movements until all 12 washers were on the two pegs in the centre of the board, and then the participant pressed the stop button. The dependent variable was total time to complete that task on the first attempt.
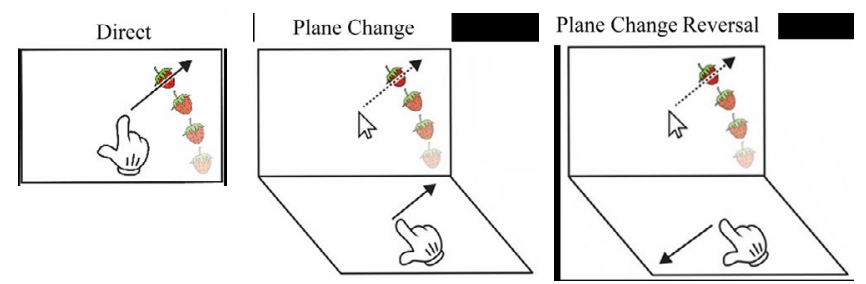

FIGURE 4. Schematic drawing of the tablet-based videogame intervention. All participants played a practice mode and a no-go inhibition mode while swiping at moving fruit on their screen to earn points across three settings: 1) Direct setting: viewing and movement plane are the same; 2) Plane Change setting: viewing and movement planes are dissociated (i.e., the player watches a vertical monitor while moving their finger on a horizontal screen); and 3) Plane Change Reversal setting: viewing and moving planes are dissociated, and the movement plane is reversed (left=right, up=down).
Multiple one-way ANOVAs indicated that there were no significant differences in age or the ratio of males and females in each group and no effects of group on baseline neurocognitive test scores. No significant differences from baseline to post-intervention in neurocognitive scores were found in any group, as expected.

\section{Progression During the Intervention Program}

Multivariate repeated-measures ANOVAs were used to assess the progression of training scores across the four months of training for at-risk and healthy training groups, according to each training condition. In the challenging mode of the training game (requiring inhibition of responses at random times), there was a significant effect of training month $(F(3,27),=$ $\left.13.742, p<.001, \eta_{\mathrm{p}}{ }^{2}=0.604\right)$ and a significant month by group interaction $\left(F(3,27)=6.789, p=.005, \eta_{\mathrm{p}}{ }^{2}=0.430\right.$; Figure 5), with significant differences between training months in the at-risk group only. In the Plane Change condition, there was only a significant effect of training month $(F(3,27)=4.454$, $p=.011, \eta_{\mathrm{p}}^{2}=0.331$; Figure 6). In the Plane Change Reversal condition, there was also an effect of training month $(F(3,27)=7.559, p=.001$; Figure 7$)$. Overall, both groups showed a significant improvement across the four months of training in five of the six conditions, with a relatively similar progression pattern.

\section{Effects of the Intervention on Fine-Motor Bimanual Tasks}

Since no assumptions of normality nor equality of error variances were violated, a univariate repeated measures ANOVA was conducted, which indicated that there was a significant effect of time point on the Bimanual Coordination Washers task $\left(F(1,13)=10.099, p=.007, \eta_{\mathrm{p}}{ }^{2}=0.437\right.$; Figure 8$)$. Notably, it was only in the at-risk training group for which LSD post hoc tests indicated that there was a significant improvement in scores $(p=.007)$ from baseline $(M=27.6 \mathrm{~s}, \mathrm{SEM}=1.477)$ to post-intervention $(M=24.6 \mathrm{~s}, \mathrm{SEM}=0.461)$. Conversely, we did not observe a significant improvement over time in the healthy training group, and there was no change in performance in the no-training groups.

\section{Change in Timing Composite Scores on the Cognitive-Motor Task Based on Training}

A multivariate repeated measures ANOVA was performed with time point as the within-subject factor, and group as the between-subject factor to assess change in the timing composite score from pre- to post-intervention across all groups. There were no significant differences in timing composites from pre- to post-intervention, nor any group by time point interactions or effects of group in any condition (Figure 9(a)).

\section{Change in End Point Error Composite Scores on the Cognitive-Motor Task Based on Training}

According to the Shapiro-Wilk tests of normality, three of the eight end point error composite score variables did not have a normal distribution; thus, non-parametric tests were used. A 
Related-Samples Wilcoxon Signed Rank Test was performed to observe each group on all four conditions for any change in median end point error composite scores from pre- to postintervention, and an independent samples Kruskal-Wallis $\mathrm{H}$ Test was used to assess differences in median scores between groups over time on each condition of this task; none of which were significant.

All of the dependent variables had equal error variances, except for the Plane Change Reversal condition at post-intervention (Levene's test: $F(3,16)=5.383, p=.009$ ).

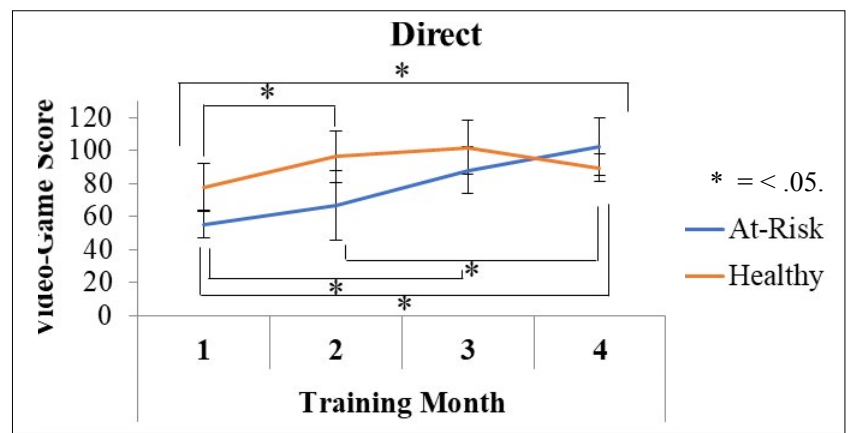

FIGURE 5. Average monthly performance on the video-game training program for both groups in the Direct condition. Error bars represent standard error of the mean (SEM).

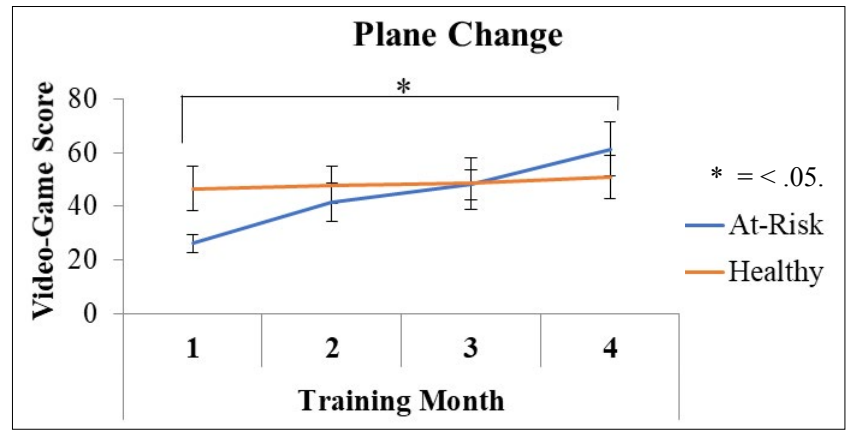

FIGURE 6. Average monthly performance on the video-game training program for both groups in the Plane Change condition. Error bars represent standard error of the mean (SEM).

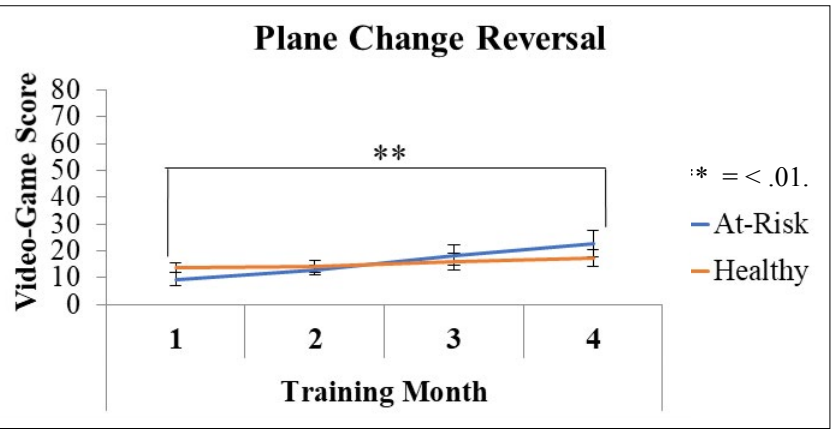

FIGURE 7. Average monthly performance on the videogame training program for both groups in the Plane Change Reversal condition. Error bars represent standard error of the mean (SEM).
To explore this further, additional univariate ANOVAs were conducted for each group to assess change in movement performance variability from pre- to post-intervention (Figure 9(b)). The resulting Levene's tests indicated that the at-risk training group had a significant reduction in variability $(F(1,8)$ $=6.371, p=.036)$ from pre- $(\mathrm{SD}=1.56)$ to post-intervention $(\mathrm{SD}=0.48)$, while the that at-risk no-training group had a non-significant increase in variability from pre- $(\mathrm{SD}=0.62)$ to post-intervention $(\mathrm{SD}=2.68)$ during this same time period. Variance did not significantly change from pre- to postintervention in either of the healthy control groups. Examples of individual trajectories demonstrating the movement performance differences as a function of group and training are presented in Figure 10.

\section{DISCUSSION}

The goal of this study was to examine the effects - if any —of an integrated cognitive-motor training exercise on functional performance in an asymptomatic population with an increased risk for developing dementia. Although cognition alone has been the focus of most assessments/interventions for dementia, cognitive ability only deteriorates after advanced disease progression, making this subjectively challenging to report. Here we focused on an objective measure of functional performance by integrating rule-based movement into the intervention. In a previous study we observed that the same training regime resulted in improved global cognition in adults in the early stages of dementia. ${ }^{(20)}$ The present study found a translation effect of visuomotor training in a cognitively healthy, but at-risk, population.

\section{Effects of the Intervention Program on Motor Performance}

The hypothesis that the at-risk group would demonstrate greater improvement over time on the measures of CMI and motor ability compared to the at-risk no-training group was partially supported. While all groups seemed to improve slightly over time, we observed an improvement post-intervention in

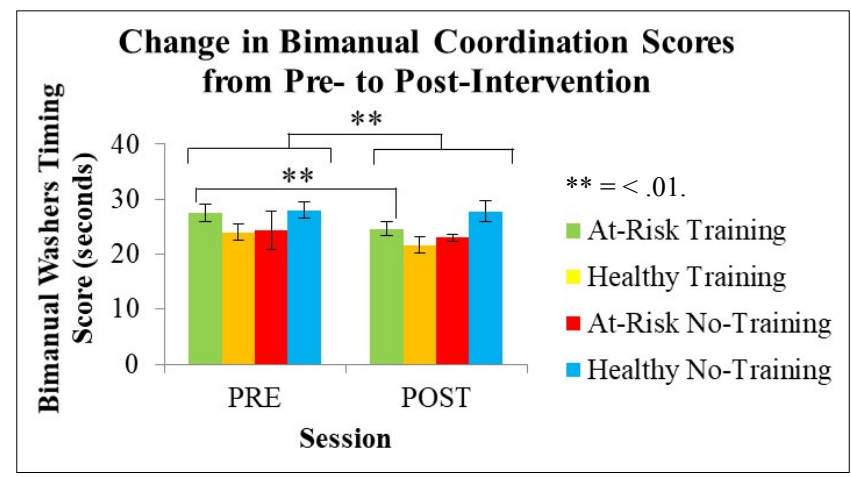

FIGURE 8. Change in Bimanual Coordination Timing scores on the washers task from baseline to the post-intervention period across each group. Error bars represent standard error of the mean (SEM). 


\section{ECHLIN: CMI TRAINING IN AT-RISK ADULTS}

bimanual coordination in the at-risk training group only. There was likely a practice effect underlying some of this improvement, generalizing from a unimanual eye-hand coordination task to a bimanual coordination task. Indeed previous studies have found some (but limited) transfer of skills between unimanual and bimanual visuomotor tasks. ${ }^{(31-32)}$ However,

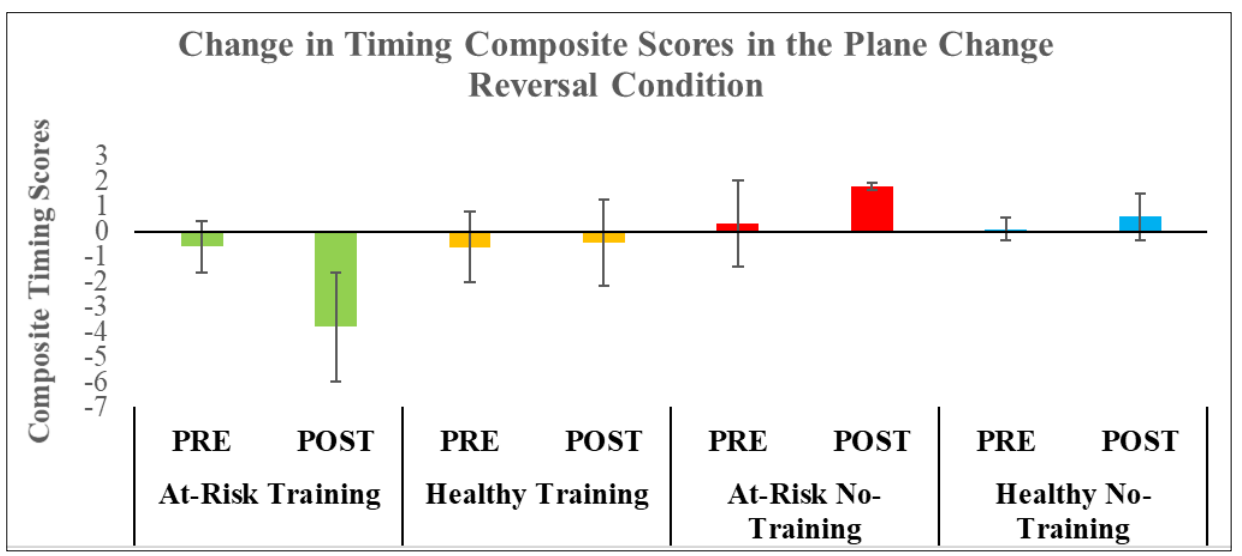

FIGURE 9. Change in the end point error composite scores during the cognitive-motor task on the Plane Change Reversal condition from baseline to the post-intervention period across each group; a negative composite score indicates better (i.e., more accurate and precise) performance; error bars represent standard error of the mean (SEM); $\rho<.05$.

\section{Healthy Participants}
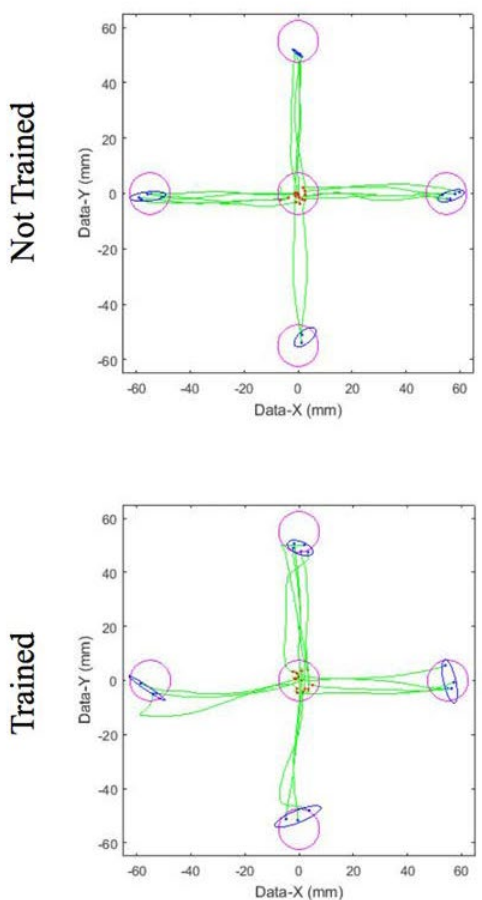

At-Risk Participants
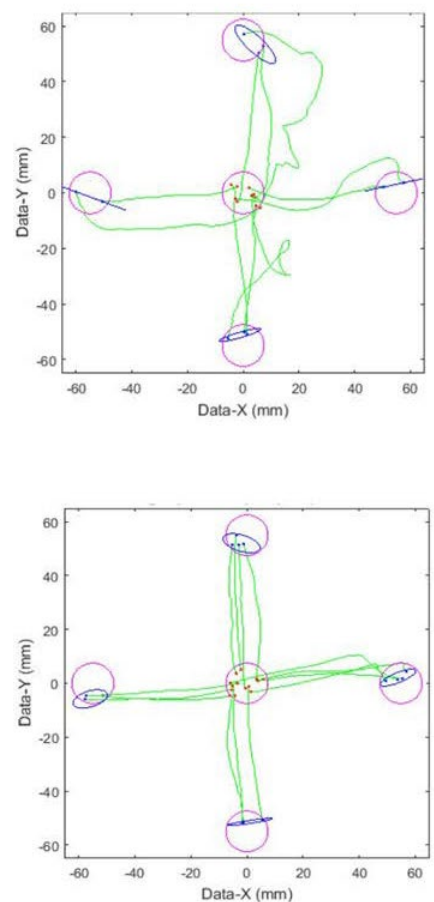

FIGURE 10. Sample hand movement trajectories from a participant in each group at post-intervention, as measured by the Plane Change Reversal condition of the Cognitive-motor task. This is the most challenging condition which involves two levels of dissociation at once. Hand trajectories began at the central target (the red dots in the central circle) and move towards one of the four peripheral targets. Each green line represents a single movement trajectory; the blue ellipses denote the $95 \%$ confidence interval for the final end point of the finger movements (the blue dots in the peripheral circles). These data provide an indication of overall cognitive-motor integration performance. A noticeable difference in overall trajectory can be seen across groups, especially in the at-risk no-training group; this participant had quite large trajectory deviations compared to the other healthy or trained groups, after an equivalent passing of time. The at-risk participant who received training appears to be performing at a similar level of ability as the healthy participants. 
practice alone cannot account for our observed improvements, given that improvement in what was likely a novel task to all participants was not observed in the healthy training group. This task required interhemispheric communication reliant on callosal fibres that transmit signals from one side of the brain to the other. Similarly, the intervention task we used here has been shown to employ large-scale neural networks that are deeply interconnected with various domains. ${ }^{(8-10)}$ Other work has found that brain regions within the frontoparietal network that are also involved with bimanual coordination ${ }^{(5)}$ show altered activation (i.e., more neural efficiency) with more experience and skill acquisition. ${ }^{(33-35)}$ Thus, while the current study's effects are subtle, we believe these data indirectly support the idea that this type of activity (controlled visuomotor exercises that integrate explicit an implicit cognitive rules for successful performance) is useful for augmenting functional brain networks shown to be impaired in those at risk for developing dementia. ${ }^{(5,15-18)}$

It is important to note that previous work on bimanual coordination has found a large sex-related difference in performance on these types of tasks ${ }^{(30,36-37)}$ and differences in visuomotor control more generally. ${ }^{(38-40)}$ While there was an overall difference in male to female ratio for our participants, we do not believe that the improvements we observed here were driven solely by the "female advantage" for bimanual tasks, since the only group to show improvement was the atrisk training group (1:4 male:female ratio) and not the healthy training group (6:6 male:female ratio) nor the no-training groups. Nevertheless, this does remain a likely contributing factor which should be accounted for by clinicians when assessing the motor abilities of female and male patients.

\section{Effects of the Intervention Program on Movement Variability}

We observed that the two groups that received training showed a significant reduction in movement variability from pre- to post-intervention on the CMI task, while the groups that did not receive training had greater movement variability at post-intervention compared to their baseline. While biological noise is an innate aspect of cellular functioning that promotes heterogeneity, sometimes having neurons in a particular system (i.e., motor systems) fire with less cohesivity may indicate a less optimal or concise signal conduction that may result in poorer motor performance. ${ }^{(41)}$ Considering that all groups in the present study were asymptomatic, we may not expect evident behavioural differences to appear on the measures tested, but the reduced movement variability across time instead suggests a reduction in noisy signals ${ }^{(42)}$ and improved efficiency of the coordination of their motor planning/execution.

\section{Baseline Motor and CMI Performance in Participants at Risk for Dementia}

The hypothesis that at-risk groups would perform worse on measures of CMI and motor ability than the healthy groups at baseline was not supported. None of the groups were significantly different on any measures at baseline testing. This finding is in contrast to earlier studies showing that at-risk females had worse CMI ability that was associated with WM disruption and reduced resting state functional connectivity. $(5,15)$ One possible reason for this lack of group differences in the present study was that, in both at-risk groups combined, half of the participants were male. Indeed, recent behavioural findings support sex-related differences in cognitive-motor behavior in at-risk adults, with males showing fewer performance deficits. ${ }^{(43)}$ Given previous findings of sex-related differences in brain activity related to cognitive-motor skill, ${ }^{(43,38-39)}$ and the combination of males and females together in the present sample may have washed-out effects observed previously for this type of task.

\section{CONCLUSIONS}

Behavioural evidence from this proof-of-principle study supports previous research indicating that cognitive-motor training may be beneficial for generalized improvement of functional ability, theoretically via increased frontoparietal network integrity. These findings have numerous therapeutic implications. Firstly, they highlight the effectiveness and simplicity of using video games to improve motor functioning. Importantly, this visuomotor training had a generalizable effect on performance for motor skills that were not directly involved in the training program itself. That is, the training program was a unimanual task, but bimanual coordination improved. This finding is consistent with previous literature suggesting that visuomotor abilities may be a more sensitive measure of early decline and effective target for intervention than measures of cognitive status. ${ }^{(5,44)}$

Secondly, since atrophy spreads across the whole brain with neurodegeneration, motor region performance should be considered for objective information about brain health. Some areas within large-scale networks are affected earlier than others; however, requiring combined output from both cognitive and motor domains increases demand on brain resources and may be a more sensitive measure of early decline. Stimulation of these networks may be producing the generalizability to other domains in our findings. ${ }^{(20)}$ Overall, these findings contribute to present knowledge about functional decline prevention in at-risk adults.

\section{ACKNOWLEDGEMENTS}

This research was undertaken as part of the Vision: Science to Applications program, thanks in part to funding from the Canada First Research Excellence Fund. This research was also supported by the York Research Chair in Brain Health and Skilled Performance (LS), and the NSERC Discovery Grant program (LS).

\section{CONFLICT OF INTEREST DISCLOSURES}

The authors declare that no conflicts of interest exist. 


\section{ECHLIN: CMI TRAINING IN AT-RISK ADULTS}

\section{APPENDICES}

APPENDIX A. Description of Cognitive-Motor Assessment Task

Four conditions of the task were used in total for each participant including direct, feedback reversal, plane change and plane change reversal conditions (see Figure 2). The task was done using a 10.1-inch tablet (ASUS Transformer Book T100 2 in 1 tablet, sampling rate: $60 \mathrm{~Hz}$ ) situated for use in the vertical plane, with an external Keytec ${ }^{\mathrm{TM}}$ touchpad (Keytec Magic Screen: Model KTMT-1315, sampling rate: $100 \mathrm{~Hz}$; Keytec $^{\text {TM }}$, Garland, TX, USA; 18 inch) placed directly below, in the horizontal plane. A calibration was done during setup so that the functional area of the Keytec ${ }^{\mathrm{TM}}$ matched the dimensions of the tablet. The Keytec ${ }^{\mathrm{TM}}$ was only used for conditions that involved a plane dissociation.

Participants sat at a table in front of this setup, where they could comfortably reach both apparatuses and were given instructions to move straight to the target as quickly and accurately as possible on all trials. The standard condition involved a direct interaction with targets on the tablet touchscreen, using the dominant hand. Participants were instructed to keep their finger on the touchscreen for the duration of the task and to move their finger to the centre of the screen.

Once the task commenced, a yellow target circle in the centre of the screen appeared $(7.5 \mathrm{~mm}$ in diameter) and participants moved their finger to this location by directly touching the screen in the vertical plane. When the custom software detected the presence of a finger in the central target, it turned green. After $2000 \mathrm{~ms}$, a red peripheral target appeared $55 \mathrm{~mm}$ away from the central target in one of four directions ( $90^{\circ}$ to the top, bottom, left or right of the centre target), eye movements were directed toward the presented target to guide the finger sliding movement on the same screen. This red circle was a cue for participants to begin their finger-sliding movement toward the peripheral target. Once the participant reached the peripheral target and remained there for $500 \mathrm{~ms}$, the peripheral target disappeared. Then, after an inter-trial interval of $2000 \mathrm{~ms}$, the yellow central home target reappeared, signaling the beginning of the next trial (see Figure 3 ). Participants completed five trials per target for a total of 20 trials per condition in random order; 80 in total.

During nonstandard conditions, all timing, presentation order and sizes/measurements were unchanged. In the feedback reversal condition, a $180^{\circ}$ visual feedback reversal was incorporated into the task, meaning that participants were required to move in the opposite direction of the intended target to successfully complete the trial; this introduces a strategic control requirement. The plane change condition involves a plane dissociation between guiding visual stimuli and hand movements. Participants were instructed to look at the targets on the vertical tablet screen, rather than look at their hand moving on the horizontal Keytec ${ }^{\text {TM }}$ touchpad below the tablet screen (i.e., gaze and hand movements were spatially dissociated from each other). The plane change reversal condition incorporated both the spatially dissociated planes between eye and hand movements, but also a $180^{\circ}$ feedback, as well. Each condition was presented in random order between participants. All nonstandard conditions required participants to keep their gaze on the vertical tablet screen throughout the task; these nonstandard conditions require CMI. Movement onset was scored as the point at which a participant's velocity surpassed $10 \%$ of their normalized peak velocity. In the same way, the movement was considered complete once velocity decelerated to lower than $10 \%$ of peak velocity within the peripheral target.

After scoring was completed, the data were processed once more to remove outliers beyond two standard deviations from the participant's mean for each outcome measure and to calculate movement variables. The kinematic outcome variables used for this study were: absolute error (AE) and variable error (VE). The AE is a measure of the distance of finger end-point position in relation to target location in millimeters, while VE represents the variability of individual finger end-point locations from the average end-point location in millimeters.

\section{REFERENCES}

1. Sperling RA, Aisen PS, Beckett LA, et al. Toward defining the preclinical stages of Alzheimer's disease: recommendations from the National Institute on Aging-Alzheimer's Association workgroups on diagnostic guidelines for Alzheimer's disease [Internet]. Alzheimer's Dement. 2011;7(3):280-92.

2. Morris J. Early-stage and preclinical Alzheimer disease. Alzheimer Dis Assoc Disord. 2005;19(3):163.

3. Ewers M, Sperling RA, Klunk WE, et al. Neuroimaging markers for the prediction and early diagnosis of Alzheimer's disease dementia [Internet]. Trends Neurosci. 2011;34(8):430-42. Available from: http://dx.doi.org/10.1016/j.tins.2011.05.005

4. Prince M, Bryce R, Albanese E, et al. The global prevalence of dementia: a systematic review and metaanalysis [Internet]. Alzheimer's Dement. 2013;9(1):63-75. Available from: http:// dx.doi.org/10.1016/j.jalz.2012.11.007

5. Hawkins KM, Goyal AI, Sergio LE. Diffusion tensor imaging correlates of cognitive-motor decline in normal aging and increased Alzheimer's disease risk. J Alzheimer's Dis. 2015; 44(3):867-78.

6. Jones DT, Knopman DS, Gunter JL, et al. Cascading network failure across the Alzheimer's disease spectrum. Brain. 2016; 139(2):547-62.

7. Clare L. Cognitive training and cognitive rehabilitation for people with early-stage Alzheimer's disease: a review. Neuropsychol Rehab. 2004;14(4)385-401.

8. Gorbet DJ, Sergio LE. Don't watch where you're going: the neural correlates of decoupling eye and arm movements. Behav Brain Res. 2016;298:229-40.

9. Gorbet DJ, Sergio LE. Looking up while reaching out: the neural correlates of making eye and arm movements in different spatial planes. Exp Brain Res. 2019;237(1):57-70.

10. Granek JA, Gorbet DJ, Sergio LE. Extensive video-game experience alters cortical networks for complex visuomotor transformations. Cortex. 2010;46(9):1165-77.

11. Salek Y, Anderson ND, Sergio L. Mild cognitive impair- 


\section{ECHLIN: CMI TRAINING IN AT-RISK ADULTS}

ment is associated with impaired visual-motor planning when visual stimuli and actions are incongruent. Eur Neurol. 2011;66(5):283-93.

12. Hawkins KM, Sergio LE. Visuomotor impairments in older adults at increased Alzheimer's disease risk. J Alzheimer's Dis. 2014;42(2):607-21.

13. Tippett WJ, Sergio LE. Visuomotor integration is impaired in early stage Alzheimer's disease. Brain Res. 2006;1102(1):92-102.

14. Tippett WJ, Krajewski A, Sergio LE. Visuomotor integration is compromised in Alzheimer's disease patients reaching for remembered targets. Eur Neurol. 2007;58(1):1-11.

15. Hawkins KM, Sergio LE. Adults at increased Alzheimer's disease risk display cognitive-motor integration impairment associated with changes in resting-state functional connectivity: a preliminary study. J Alzheimer's Dis. 2016;53(3):1161-72.

16. Fischer FU, Wolf D, Scheurich A, et al. Altered whole-brain white matter networks in preclinical Alzheimer's disease [Internet]. NeuroImage Clin. 2015;8:660-66.

17. Honea RA, Swerdlow RH, Vidoni ED, et al. Progressive regional atrophy in normal adults with a maternal history of Alzheimer disease. Neurology. 2011;76(9):822-29.

18. Mosconi L, Brys M, Switalski R, et al. Maternal family history of Alzheimer's disease predisposes to reduced brain glucose metabolism [Internet]. Proc Natl Acad Sci USA. 2007; 104(48):19067-72.

19. Voineskos AN, Rajji TK, Lobaugh NJ, et al. Age-related decline in white matter tract integrity and cognitive performance: a DTI tractography and structural equation modeling study. Neurobiol Aging. 2012;33(1):21-34.

20. de Boer C, Echlin HV, Rogojin A, et al. Thinking-while-moving exercises may improve cognition in elderly with mild cognitive deficits: a proof-of-principle study. Dement Geriatr Cogn Dis Extra. 2018;8:248-58.

21. Tippett WJ, Rizkalla MN. Brain training: rationale, methods, and pilot data for a specific visuomotor/visuospatial activity program to change progressive cognitive decline. Brain Behav. 2014;4(2):171-79.

22. Elble RJ, Leffler K. Pushing and pulling with the upper extremities while standing: the effects of mild Alzheimer dementia and Parkinson's disease. Mov Disord. 2000;15(2):255-68.

23. Mosconi L, Glodzik L, Mistur R, et al. Oxidative stress and amyloid-beta pathology in normal individuals with a maternal history of Alzheimer's [Internet]. Biol Psychiatry. 2010; 68(10):913-21.

24. Schmidt R, Kienbacher E, Benke T, et al. Sex differences in Alzheimer's disease [in German]. Neuropsychiatrie. 2008; 22(1):1-15.

25. Duara R, Barker W, Lopez-Alberola R, et al. Alzheimer's disease: interaction of apolipoprotein $\mathrm{E}$ genotype, family history of dementia, gender, education, ethnicity, and age of onset. Neurology. 1996;46(6):1575-79.

26. Fratiglioni L, Ahlbom A, Viitanen M, et al. Risk factors for lateonset Alzheimer's disease: A population-based, case-control study . Ann Neurol. 1993;33:258-66. Cited 2018 Oct 28.

27. Green RC, Cupples A, Go R, et al. Risk of dementia among white and African American relatives of patients with alzheimer disease [Internet]. JAMA. 2002;287(3):329-36. Available from: http://jama.jamanetwork.com/article.aspx?doi=10.1001/ jama.287.3.329
28. Reitz C, Mayeux R. Alzheimer disease: epidemiology, diagnostic criteria, risk factors and biomarkers [Internet]. Biochem Pharmacol. 2014;88(4):640-51

29. Johnson-Greene D. Dementia Rating Scale-2 (DRS-2) by P.J. Jurica, C.L. Leitten, and S. Mattis: Psychological Assessment Resources, 2001 [Internet]. Arch Clin Neuropsychol. 2004; 19(1):145-47. Available from: http://www.sciencedirect.com/ science/article/pii/S0887617703001148

30. Albines D, Granek J, Gorbet G, et al. Bimanual coordination development is enhanced in young females and experienced athletes. J Mot Learn Dev. 2016;4(2):274-86.

31. Nozaki D, Kurtzer I, Scott SH. Limited transfer of learning between unimanual and bimanual skills within the same limb. Nat Neurosci. 2006;9(11):1364-66.

32. Wang J, Sainburg RL. Generalization of visuomotor learning between bilateral and unilateral conditions. J Neurophysiol. 2009;102(5):2090-99.

33. Haslinger B, Erhard P, Altenmüller E, et al. Reduced recruitment of motor association areas during bimanual coordination in concert pianists. Hum Brain Mapp. 2004;22(3):206-15.

34. Jäncke L, Shah NJ, Peters M. Cortical activations in primary and secondary motor areas for complex bimanual movements in professional pianists. Cogn Brain Res. 2000;10(1-2):177-83.

35. Sun FT, Miller LM, Rao AA, et al. Functional connectivity of cortical networks involved in bimanual motor sequence learning. Cereb Cortex. 2007;17(5):1227-34.

36. Denckla M. Development of speed in repetitive and successive finger-movements in normal children. Devel Med Child Neurol. 1973;15(5):635-45.

37. Allen L, Richey M, Chai Y, et al. Sex differences in the corpus callosum of the living human being. JNeurosci. 1991;11(4):933-42.

38. Gorbet DJ, Sergio LE. Preliminary sex differences in human cortical BOLD fMRI activity during the preparation of increasingly complex visually guided movements. Eur J Neurosci. 2007;25(4):1228-39.

39. Gorbet DJ, Mader LB, Staines WR. Sex-related differences in the hemispheric laterality of slow cortical potentials during the preparation of visually guided movements. Exp Brain Res. 2010;202(3):633-46.

40. Gorbet DJ, Staines WR. Inhibition of contralateral premotor cortex delays visually guided reaching movements in men but not in women. Exp Brain Res. 2012;212(2):315-25.

41. Matthews PBC. Relationship of firing intervals of human motor units to the trajectory of post-spike after-hyperpolarization and synaptic noise. $J$ Physiol. 1996;492(2):597-628.

42. Müller H, Sternad D. Decomposition of variability in the execution of goal-oriented tasks: three components of skill improvement. J Exp Psychol Hum Percept Perform. 2004;30(1):212-33.

43. Rogojin A, Gorbet DJ, Hawkins KM, et al. Cognitive-motor integration performance is affected by sex, APOE status, and family history of dementia. J Alzheimers Dis. 2019;71(2):685-701.

44. Buchman AS, Bennett DA. Loss of motor function in preclinical Alzheimer's disease. Expert Rev Neurother. 2011;11(5):665-76.

Correspondence to: Lauren Sergio, PhD, School of Kinesiology and Health Science, York University, 357 Bethune Collage, 4700 Keele St., North York, ON, M3J 1P3 Canada E-mail: lsergio@yorku.ca 